\title{
Frequency of fever episodes related to febrile seizure recurrence
}

\author{
M van Stuijvenberg ${ }^{1}$, NE Jansen $^{1}$, EW Steyerberg ${ }^{2}$, G Derksen-Lubsen $^{3}$ and HA Moll ${ }^{1}$ \\ Department of Paediatrics ${ }^{1}$, Sophia Children's Hospital, Rotterdam; ${ }^{2}$ Department of Public Health, Erasmus University, Rotterdam; \\ ${ }^{3}$ Department of Paediatrics, Juliana Children's Hospital, Den Haag, The Netherlands
}

\begin{abstract}
Stuijvenberg M van, Jansen NE, Steyerberg EW, Derksen-Lubsen G, Moll HA. Frequency of fever episodes related to febrile seizure recurrence. Acta Pædiatr 1999; 88: 52-5. Stockholm. ISSN 0803-5253

The aim of this study was to assess the number of fever episodes as a risk factor for febrile seizure recurrence during the first 6 months after the last previous febrile seizure. In a 6-month follow-up study of 155 children, aged 3 months to $5 \mathrm{y}$, with a first or a recurrent febrile seizure, the occurrence of fever episodes and febrile seizure recurrences was prospectively documented. Using logistic regression analysis the association between the baseline characteristics and the number of fever episodes and the outcome, a febrile seizure recurrence, was studied. In total, 260 fever episodes were registered; 29 children experienced 1 or more febrile seizure recurrence during follow-up. Two factors were associated with febrile seizure recurrence: the number of fever episodes [odds ratio $(\mathrm{OR})=1.8 ; 95 \%$ confidence interval $(\mathrm{CI}): 1.4-2.4)$ ] and age at study entry $(\mathrm{OR}=0.6 ; 95 \% \mathrm{CI}: 0.3-1.1)$. In a multivariable model, only the number of fever episodes remained significant. In conclusion, the number of fever episodes increases the risk of a febrile seizure recurrence with a factor of 1.8 per fever episode in the first 6 months after a febrile seizure. $\square$ Febrile seizure recurrence, logistic regression, number of fever episodes
\end{abstract}

HA Moll, Sophia Children's Hospital, Room Sp 1543, Dr Molewaterplein 60, 3015 GJ Rotterdam, The Netherlands)

Febrile seizures are the most common type of seizures in children aged 3 months to $5 \mathrm{y}$, with a cumulative incidence of $4 \%(1,2)$. The 2 -y probability of a febrile seizure recurrence has been estimated as $30 \%$ on average; $10-20 \%$ of the seizures recur within 6 months, with a decreasing risk after the first 6 months following the previous seizure $(3,4)$.

The risk of febrile seizure recurrences has been studied thoroughly (3-9). The major and most consistent risk factors are: young age at onset, family history of febrile seizures, previous recurrent febrile seizures, a low temperature at the initial febrile seizure, a multiple type initial febrile seizure and short time lapse since the previous seizure.

In addition, it has been demonstrated that frequent fever episodes are associated with an increased risk of recurrence $(9,10)$. Children have the highest risk of recurrence in the first 6 months after the last febrile seizure. This study aimed to assess the incidence of fever episodes in relation to febrile seizure recurrence during this high-risk period for febrile seizure recurrence.

\section{Patients and methods}

\section{Patients}

Children aged 3 months to $5 \mathrm{y}$, who were seen for an initial or a recurrent febrile seizure at the Sophia
Children's Hospital in Rotterdam and the Juliana Children's Hospital in The Hague between 1 April 1994 and 1 July 1997, were considered for inclusion. Both outpatients' clinics include mainly basic specialist care. Eligible children were enrolled either in a randomized placebo-controlled trial of ibuprofen syrup to prevent febrile seizure recurrences (1994-1996), of which the results will be reported elsewhere, or in a cohort study, in which the occurrence of fever episodes and febrile seizure recurrence during 6 months was registered (1996-1997). The definition of febrile seizure of the National Institutes of Health Consensus Meeting (2) was used. The parents of all participating children gave their written informed consent. The study protocol was approved by the relevant local ethical review boards.

Additional inclusion criteria for the children in the randomized controlled trial were: aged between 1 and 4 $\mathrm{y}$; presence of one or more risk factors for seizure recurrence, defined as: a positive first-degree family history of febrile seizures; an initial seizure of the multiple type; a temperature below $40^{\circ} \mathrm{C}$ at the initial febrile seizure; and one or more previous febrile seizure recurrences.

Exclusion criteria for the present study were: more than 2 months' time lapse between the last previous febrile seizure and study entry; current use of antiepileptic drugs; no telephone connection; non-Dutchand non-English-speaking parents. The randomized 
controlled trial included the children who were allocated to placebo only.

\section{Methods}

After the children had visited the emergency room of the hospital with a febrile seizure, they received an appointment for special febrile seizure outpatients' clinic hours, where they were examined and the parents were given information regarding fever and febrile seizures. The parents were advised against giving their child antipyretics during fever.

Baseline characteristics were recorded and checked at study entry, including age, gender, family history of febrile seizures and epilepsy, date and characteristics of the initial febrile seizure, the number of previous recurrent febrile seizures and the date of the last previous febrile seizure. The temperature and the presence of complex characteristics during the initial febrile seizure were documented. Complex characteristics were defined as seizure duration of $15 \mathrm{~min}$ or more, recurrence of the seizure within $24 \mathrm{~h}$ (multiple type), and/or a focal onset of the seizure or a postictal Todd's paresis of the facial muscles or limbs (focal characteristics) $(11,12)$.

If parents consented to participate in the randomized controlled trial, they were instructed to take the rectal temperature of the child immediately when the child seemed ill or feverish and to start promptly to administer the study medication when the temperature was $38.5^{\circ} \mathrm{C}$ or higher. They were instructed to record the precise timing of administering of the study medication and the height of the temperature on a standardized patient form and to notify the investigator of each fever episode and recurrent febrile seizure. If no fever had been reported after 3 months, the investigator contacted the parents to ensure participation and to check the occurrence of fever or febrile seizure recurrence. When a febrile seizure recurrence occurred, the follow-up time was considered as terminated for the randomized trial; however, the parents were asked to continue to list all subsequent fever episodes and seizure recurrences until the planned date of study termination (October 1996).

Parents who consented to participate in the cohort study were given six diaries. They were instructed to use subsequently one diary per month of follow-up to register the occurrence of fever episodes and recurrent febrile seizures. The parents were instructed to document every day whether or not their child was feverish. When the child seemed ill or feverish, the parents took the child's rectal temperature; when it was $38.5^{\circ} \mathrm{C}$ or more, they were asked to mark that day with 'yes' and when the rectal temperature was lower than $38.5^{\circ} \mathrm{C}$, with 'no'. Accordingly, the occurrence of one or more febrile seizure recurrences was documented. After every month of follow-up, the parents sent the diary to the investigator. If the diary was not received promptly or if data were not filled in, the parents were contacted to ensure participation and to check the occurrence of fever or febrile seizure recurrence.

\section{Statistical analysis}

The outcome was defined as the occurrence of a febrile seizure recurrence within the 6-month follow-up period. If the follow-up time was terminated before 6 months, children were excluded from the analysis. Early termination of follow-up was defined as: development of afebrile seizures; starting the use of intermittent prophylactic diazepam; and refusal to participate further in the study.

Logistic regression analysis was used to assess the association between baseline characteristics, the number of fever episodes and the outcome. Baseline characteristics included the following risk factors for febrile seizure recurrence: a first-degree family history of febrile seizures, age at onset $<1 \mathrm{y}$, temperature at the initial febrile seizure $<40^{\circ} \mathrm{C}$, multiple type initial febrile seizure and one or more previous recurrent febrile seizures. All characteristics with $p<0.10$ in the univariable analysis were entered in a multivariable model, to adjust for their interrelation. Odds ratios (OR) were used as a measure of association. Associations were statistically significant $(p<0.05)$, if the $95 \%$ confidence interval $(95 \% \mathrm{CI})$ of the OR did not include the value 1. SPSS for Windows was used for the analysis.

To assess any difference between the randomized controlled trial group and the cohort study group, univariable regression analyses were performed separately in each group. If different OR for baseline characteristics were found, a study-interaction term, indicating the original study (randomized controlled trial or cohort study), was introduced into the model and multiplied by the baseline characteristics involved, to assess whether differences were likely to be due to chance.

\section{Results}

\section{Study population}

The study population consisted of 171 children; 89 (52\%) children allocated to the use of placebo in the randomized controlled trial and $82(48 \%)$ in the cohort study. Sixteen children of the cohort study were excluded from the analysis, because of early termination. Of these, 2 children developed afebrile seizures, 1 child received intermittent prophylactic treatment with diazepam and 13 parents refused participation after informed consent had initially been given. Thus, the data from 155 children were used for the analysis; 89 (57\%) children from the randomized controlled trial and $66(43 \%)$ from the cohort study. 
Table 1. Baseline characteristics, fever episodes and febrile seizure recurrence.

\begin{tabular}{|c|c|c|c|c|c|c|}
\hline & \multirow{2}{*}{$\begin{array}{c}\text { Recurrence } \\
\text { during follow-up } \\
n=29 \\
(100 \%)\end{array}$} & \multirow{2}{*}{$\begin{array}{l}\text { No recurrence } \\
\text { during follow-up } \\
n=126 \\
(100 \%)\end{array}$} & \multirow{2}{*}{$\begin{array}{c}\text { Univariable } \\
\text { analysis } \\
\text { OR }(95 \% \mathrm{CI})\end{array}$} & \multicolumn{2}{|c|}{ Multivariable analysis } & \multirow[b]{2}{*}{$p$-value } \\
\hline & & & & $p$-value & OR $(95 \% \mathrm{CI})$ & \\
\hline \multicolumn{7}{|l|}{ Baseline characteristics } \\
\hline Female gender & $13(45 \%)$ & $43(34 \%)$ & $1.6(0.7-3.6)$ & 0.28 & - & - \\
\hline Age at study entry (in years) ${ }^{a}$ & $1.6(1.3-2.1)$ & $1.8(1.4-2.6)$ & $0.6(0.3-1.1)$ & 0.09 & $1.0(0.5-1.8)$ & 0.89 \\
\hline Number of febrile episodes ${ }^{\mathrm{a}}$ & $2.0(2.0-4.0)$ & $1.0(0.0-2.0)$ & $1.8(1.4-2.4)$ & $<0.001$ & $1.8(1.4-2.4)$ & $<0.001$ \\
\hline
\end{tabular}

\section{Febrile seizure recurrence during follow-up}

Of the 155 children, 29 (19\%) children had 1 or more febrile seizure recurrences and $126(81 \%)$ had no febrile seizure recurrence during the 6-month follow-up period. In total, 41 febrile seizures occurred; 20 children suffered 1 recurrence, 6 children had 2 recurrences and 3 children had 3 recurrences.

A total of 260 fever episodes was counted in 155 children and the mean number of fever episodes per child was 1.7. Of all 155 children, 42 (27\%) had no fever episodes and $113(73 \%)$ had 1 or more fever episodes. Among those 113, 29 children had a febrile seizure recurrence with 89 fever episodes (mean number of fever episodes per child 3.1, median 2.0). The 126 children without a recurrence had 171 fever episodes (mean 1.4, median 1.0).

As shown in Table 1, in the univariable analysis age at study entry $(\mathrm{OR}=0.6)$ and number of fever episodes $(\mathrm{OR}=1.8)$ were associated with febrile seizure recurrence $(p<0.10)$. The other baseline characteristics, including the risk factors for febrile seizure recurrence, were not associated with febrile seizure recurrence. In a multivariable model, the number of febrile episodes was significantly associated with febrile seizure recurrence. The risk of a febrile seizure recurrence was increased by a factor of 1.8 per fever episode in the first 6 months after the last previous febrile seizure. Age at study entry had no additional effect on the outcome in the multivariable model $(\mathrm{OR}=1.0)$. Children at a young age experienced more fever episodes than older children (Fig. 1). From Fig. 1 it can be seen that children with a recurrence during follow-up experienced more fever episodes than the other children. For a given number of fever episodes, the risk of recurrence shows no clear association with age. Children at a young age had more recurrences owing to the high number of fever episodes in young children.

In separate analyses of the randomized controlled trial and the cohort study, no statistically significant differences were found in the OR of baseline characteristics with febrile seizure recurrence. The separate probabilities of febrile seizure recurrence in the 6month follow-up period were $21 \%$ in the randomized controlled trial and $15 \%$ in the cohort study $\left(\chi^{2}\right.$, $p=0.33)$.

\section{Discussion}

This study demonstrates that the number of fever episodes was a factor independently associated with a febrile seizure recurrence in the first 6 months after the last previous febrile seizure. The risk of a febrile seizure recurrence was increased by a factor of 1.8 per fever episode; children with febrile seizures who suffer frequently from feverish illnesses have a high risk of recurrence. Two follow-up studies of children who had had an initial febrile seizure found similar results $(9,10)$. Rantala and Uhari evaluated retrospectively the frequency of fever episodes and febrile seizure recurrences, using a mailed questionnaire. After a mean follow-up period of $3.8 \mathrm{y}$, they found that the number of fever episodes was a significant risk factor for the recurrence of febrile seizures. Unfortunately, no quantification of the relative risk was reported (9). Knudsen demonstrated that in the year after an initial febrile seizure, children who suffer few fever episodes (defined as fewer than $4 \mathrm{y}^{-1}$ ) had 0.06 times the risk of febrile seizure recurrence of children suffering 4 or more fever episodes per year (OR 1:16) (10). Concordantly, when the number of fever episodes was categorized in the

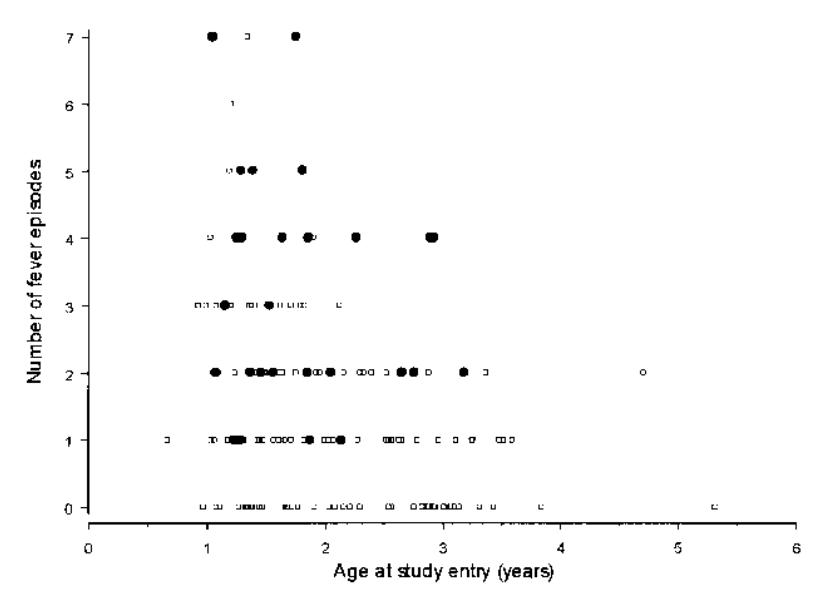

Fig. 1. Number of fever episodes in relation to the age at study entry and febrile seizure recurrence. $\square$ : Cases without a febrile seizure recurrence during follow-up; 0 : cases with a febrile seizure recurrence during follow-up. 
present study (fewer than 2 episodes vs 2 or more episodes per 6 months) a comparable risk reduction of 0.09 was found in the group of children with fewer than 2 fever episodes per 6 months.

In the present study the mean number of fever episodes per child was 1.7 in 6 months of follow-up. Children with 1 or more febrile seizure recurrences had a mean of 3.1 , compared with 1.4 in children who experienced no febrile seizure recurrence, illustrating a higher frequency of febrile episodes in children with febrile seizure recurrence. In other febrile seizure studies, the mean number of fever episodes per child per year has been reported as 3.0 (10) or was calculated from the presented data as $1.5-1.9(9,13,14)$. All of these studies had a median follow-up time longer than 6 months. The relatively high estimate of 1.7 fever episodes per 6 months in the present study is probably due to the fact that the risk over time may not be constant, since increasing age decreases the incidence (Fig. 1) (10).

The combination of the data from the randomized controlled trial and the cohort study is justified, because both are prospective follow-up studies which were carried out in the same hospitals, by the same investigators, within a ongoing study period of $3 \mathrm{y}$ (1994-1997). All parents had received similar instructions regarding the use of antipyretics and children had not received a standardized dose of ibuprofen during fever. All children had a similar follow-up time at risk of 6 months. Further separate analysis of the two original studies showed no difference with respect to the association of baseline characteristics with febrile seizure recurrence.

The 6-month probabilities of febrile seizure recurrence of both studies were reported separately (21\% and $15 \%$, respectively). The fact that the children who had been participating in the randomized controlled trial had one or more risk factors at study entry may explain the slightly higher risk of recurrence. Further, the risk of recurrence of $15 \%$ per 6 months in the cohort study is in accordance with previously reported average probabilities of $10-20 \%(3,4)$.

The association between the number of fever episodes and seizure recurrence may suggest that the factor 'chance' plays an important role in the pathophysiology of febrile seizures. The more fever episodes a child experiences, the higher the probability of another recurrent seizure, independent of other risk factors of febrile seizure recurrence.

The results of this study may have consequences for the provision of information to parents. If a child usually suffers from frequent fever episodes, the parents may be informed of a probable increased risk of seizure recurrence in the first 6 months after a febrile seizure. If intermittent preventive treatment (i.e. diazepam during fever, $1 \mathrm{mg} \mathrm{kg}$ body weight ${ }^{-1} \mathrm{~d}^{-1}$, given in 3 doses) is considered necessary, because of a very high risk of recurrence or very frightened parents, the frequency of fever episodes may play an additional role in this decision (14). However, parents may also be reassured because the number of fever episodes will decrease as the child grows older; thus, the risk of febrile seizure recurrence will decrease accordingly.

\section{Conclusion}

In conclusion, the number of fever episodes is independently associated with febrile seizure recurrence. During the first 6 months after a febrile seizure the risk of recurrence is increased by a factor of 1.8 per fever episode.

\section{References}

1. Offringa M, Hazebroek-Kampschreur AAJM, Derksen-Lubsen G. Prevalence of febrile seizures in Dutch schoolchildren. Paediatr Perinat Epidemiol 1991; 5: 181-8

2. National Institutes of Health Consensus Statement. Febrile seizures. Pediatrics 1980; 66: 1009-12

3. Offringa M, Bossuyt PMM, Lubsen J, Ellenberg JH, Nelson KB, Knudsen FU, et al. Risk factors for seizure recurrence in children with febrile seizures: a pooled analysis of individual patient data from five studies. J Pediatr 1994; 124: 572-84

4. Offringa M, Derksen-Lubsen G, Bossuyt PMM, Lubsen J. Seizure recurrence after a first febrile seizure: a multivariate approach. Dev Med Child Neurol 1992; 34: 15-24

5. El-Radhi AS, Banajeh S. Effect of fever on recurrence rate of febrile convulsions. Arch Dis Child 1989; 64: 869-70

6. Esch A, van Steyerberg EW, Berger MY, Offringa M, DerksenLubsen G, Habbema JDF. Family history and recurrence of febrile seizures. Arch Dis Child 1994; 70: 395-9

7. Rantala H, Uhari M, Tuokko H. Viral infections and recurrences of febrile convulsions. J Pediatr 1990; 116: 195-9

8. Berg AT, Shinnar S, Hauser WA, Alemany M, Shapiro ED, Salomon ME, Crain EF. A prospective study of recurrent febrile seizures. N Engl J Med 1992; 327: 1122-7

9. Rantala H, Uhari M. Risk factors for recurrences of febrile convulsions. Acta Neurol Scand 1994; 90: 207-10

10. Knudsen FU. Frequent febrile episodes and recurrent febrile convulsions. Acta Neurol Scand 1988; 78: 414-7

11. Berg AT, Steinschneider M, Kang H, Shinnar S. Classification of complex features of febrile seizures: interrater agreement. Epilepsia 1992; 33: 661-6

12. , Commission on Epidemiology and Prognosis of the International League against Epilepsy. Guidelines for epidemiologic studies on epilepsy. Epilepsia 1993; 34: 592-6

13. Uhari M, Rantala H, Vainiopää L, Kurttila R. Effect of acetaminophen and of low intermittent doses of diazepam on prevention of recurrences of febrile seizures. J Pediatr 1995; 126: 991-5

14. Rosman PN, Colton T, Labazzo J, Gilbert PL, Gardella NB, Kaye EM, et al.. A controlled trial of diazepam administered during febrile illnesses to prevent recurrence of febrile seizures. N Engl J Med 1993; 329: 79-84

Received May 11, 1998. Accepted in revised form Aug. 4, 1998 